\title{
ACOUSTICS2008/2106 \\ The simulation of bat-oriented auditory processing using the experimental data of echolocating signals
}

\author{
S.Y. Kim, R. Allen and D. Rowan \\ University of Southampton, SPCG-ISVR, SO17 1BJ Southampton, UK
}

\begin{abstract}
There are various approaches to understanding the echolocation phenomenon of bats. A part of the echolocating process is assessed here by determining what acoustic signal a bat's ears receive during echolocation. It is simplified in an experimental rig to measure the reflections from objects in different horizontal angles which represents a sound localisation task in bats. It has been assumed in this study that the remarkable echolocating ability of bats, which is not shown in the most other mammalian species, is achieved by their different physical shape of head and ears, and specialised auditory processing of echolocating signals. In human studies in terms of sound localisation, physical characteristics are usually modelled as a head-related transfer function (or HRTF) and gammatone filter banks are widely used to simulate auditory processing in the cochlear. A modified filterbank is used here to represent the auditory processing in bats and combined with the experimental data of object reflections. Bat HRTFs will be used subsequently to determine the acoustic reflections at both ears.
\end{abstract}

Keywords:

Technical area: Animal Bioacoustics (AB)

PACS \#1: 43.80.Ka Sound production by animals: mechanisms, characteristics, populations, biosonar (see also 43.30.Nb and 43.64.Tk)

PACS \#2: 43.80.Lb Sound reception by animals: anatomy, physiology, auditory capacities, processing (see also 43.64.Tk, 43.66.Gf)

PACS \#3: 